APS

physics

This is the accepted manuscript made available via CHORUS. The article has been published as:

\title{
Longitudinal Spin Relaxation of Optically Pumped Rubidium Atoms in Solid Parahydrogen
}

Sunil Upadhyay, Andrew N. Kanagin, Chase Hartzell, Tim Christy, W. Patrick Arnott, Takamasa Momose, David Patterson, and Jonathan D. Weinstein

Phys. Rev. Lett. 117, 175301 - Published 20 October 2016

DOI: 10.1103/PhysRevLett.117.175301 


\title{
Longitudinal spin relaxation of optically pumped rubidium atoms in solid parahydrogen
}

\author{
Sunil Upadhyay, ${ }^{1}$ Andrew N. Kanagin, ${ }^{1}$ Chase Hartzell, ${ }^{1}$ Tim Christy ${ }^{1}$ W. Patrick \\ Arnott, ${ }^{1}$ Takamasa Momose, ${ }^{2}$ David Patterson, ${ }^{3}$ and Jonathan D. Weinstein ${ }^{1, *}$ \\ ${ }^{1}$ Department of Physics, University of Nevada, Reno NV 89557, USA \\ ${ }^{2}$ Department of Chemistry, University of British Columbia, \\ 2036 Main Mall Vancouver, BC, 16 T 1Z1, Canada \\ ${ }^{3}$ Department of Physics, Harvard University, Cambridge MA 02138, USA
}

\begin{abstract}
We have grown crystals of solid parahydrogen using a single closed-cycle cryostat. We have doped the crystals with rubidium atoms at densities on the order of $10^{17} \mathrm{~cm}^{-3}$ and used optical pumping to polarize the spin state of the implanted atoms. The optical spectrum of the rubidium atoms shows larger broadening than previous work in which the rubidium was implanted in solid argon or neon. However, the optical pumping behavior is significantly improved, with both a larger optical pumping signal and a longer longitudinal relaxation time. The spin relaxation time shows a strong dependence on orthohydrogen impurity levels in the crystal, as well as the applied magnetic field. Current performance is comparable to state-of-the-art solid state systems at comparable spin densities, with potential for improvement at higher parahydrogen purities.
\end{abstract}

PACS numbers: 32.80.Xx, 32.30.-r, 07.55.Ge

Experimental systems with localized electron spins are of interest for applications such as qubits [1] and magnetic sensors [2-4], for exploring many-body physics [5], and for fundamental physics experiments [6]. Key performance requirements for these applications are the ability to efficiently prepare and detect the spin states, and the ability to achieve long coherence times $\left(\mathrm{T}_{2}\right)$ at high densities.

A promising, relatively unexplored physical system for achieving those criteria is atoms implanted in a cryogenic parahydrogen matrix. In this work, we investigate rubidium atoms implanted in parahydrogen at densities on the order of $10^{17} \mathrm{~cm}^{-3}$. We demonstrate the ability to prepare and detect the Rb spin states, and measure the longitudinal relaxation time of the spin $\left(T_{1}\right) . T_{1}$ is important because it provides a fundamental limit on the coherence time (although we note that many systems fall short of that limit) $[7,8]$.

There are many other systems attempting to attain these same goals. For example, gas phase atoms and ions may be localized in magnetic, electric, or optical traps. While these systems can achieve excellent coherence times, they are currently unable to attain comparably high densities. The only systems capable of achieving such high densities are those that "trap" spins in the solid phase. Two well-developed solid-phase systems are NV centers [1] and phosphorous donors in silicon [9]. The spin state of NV centers may be optically prepared and measured with high efficiency. For cryogenic samples with NV densities of $5 \times 10^{17} \mathrm{~cm}^{-3}, \mathrm{~T}_{1} \approx 17 \mathrm{~s}$ [7]. Phosphorous donors in silicon have shown both high efficiency readout and electron spin relaxation times of $\mathrm{T}_{1} \sim 3 \times 10^{3} \mathrm{~s}$ at low densities and cryogenic temperatures $[8,10]$. However, the relaxation time degrades rapidly with increasing density, and drops to $\mathrm{T}_{1} \sim 1 \mathrm{~s}$ at densities of $10^{17} \mathrm{~cm}^{-3}[9,10]$.

We note that longer relaxation times and coherence times may be achieved at even higher densities when employing nuclear spins: rare-earth ions in cryogenic crystals exhibit decoherence times on the order of $10^{2} \mathrm{~s}$ at densities of $10^{19} \mathrm{~cm}^{-3}$ [11, 12]; liquid ${ }^{129} \mathrm{Xe}$ has longitudinal and transverse relaxation times on the order of $10^{3} \mathrm{~s}$ at densities of $10^{22} \mathrm{~cm}^{-3}[13]$.

Another promising route for achieving high densities of addressable electron spins are matrix isolation experiments, where atoms or molecules of interest are trapped inside a cryogenic matrix of an inert material. Traditionally, noble gases are chosen for this role. High densities of dopant atoms can be achieved, and because of the weak interaction with the host matrix, the dopant atoms retain many of the favorable properties of gas-phase atoms [14].

Optical pumping of the spin state of rubidium atoms in a solid argon matrix was previously demonstrated [4]. However, $\mathrm{T}_{1}$ times were not competitive with NV centers, and polarization signals were small, indicating that either state preparation or readout (or some combination of the two) was poor. Rubidium atoms implanted in solid neon demonstrated even worse behavior [15]. Much better results were obtained for Cs atoms in solid helium, where optical pumping and readout was significantly more efficient, and $\mathrm{T}_{1}$ times of $\sim 2 \mathrm{~s}$ were observed $[16,17]$. However, no one has yet been able to achieve high densities of implanted alkali atoms in solid helium [18].

In this work, we show that Rb atoms implanted in parahydrogen are able to achieve polarization signals and $\mathrm{T}_{1}$ times roughly one order of magnitude higher than what was accomplished in argon. Surprisingly, $\mathrm{T}_{1}$ times superior to that of alkali atoms in either solid $[16,17]$ or superfluid [19] helium were observed at densities many 
orders of magnitude higher. Parahydrogen shows results competitive with what has been achieved with NV centers and phosphorous donors in silicon, with the potential for improvement and the possibility of working with a wide variety of dopant atoms.

We grow our samples by co-condensing parahydrogen and atomic rubidium on a cold substrate, as described in the Supplemental Material [20], which includes Refs. [21-28]. The fraction of orthohydrogen impurities is $\lesssim$ $1 \%$. An optical spectrum of a typical crystal is shown in Fig. 1; the section doped with rubidium was $1 \mathrm{~mm}$ thick. From the absorption spectrum in Fig. 1, we determine the $\mathrm{Rb}$ density to be $1 \times 10^{17} \mathrm{~cm}^{-3}$.

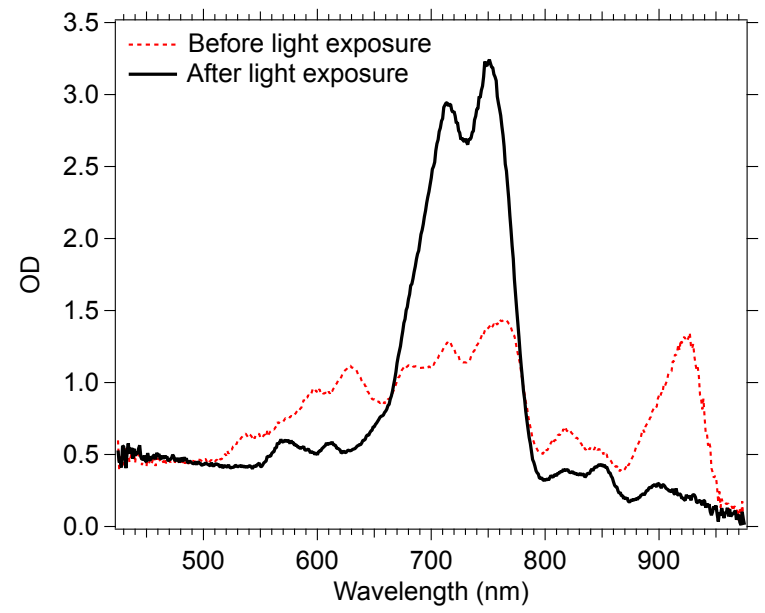

FIG. 1. (Color online) Spectra of rubidium-doped parahydrogen crystals, before and after exposure to broadband light, as described in the text. Both spectra show enormous broadening when compared to gas-phase $\mathrm{Rb}$ atoms, which has two strong, narrow absorption lines at 780 and $795 \mathrm{~nm}$. The transmission $T$ of the crystal is determined by comparing spectra before and after crystal deposition; $T \equiv e^{-\mathrm{OD}}$.

Two spectra are shown in Fig. 1. Both were taken at the base temperature of our cryostat, two days after crystal growth. In the first spectrum, the crystal had minimal exposure to light during and after its growth. This spectrum was similar to the spectrum immediately following crystal growth, indicating that changes to the spectrum in the absence of applied light are slow or nonexistent. The second spectrum shows the same crystal after exposure to broadband light from a halogen lamp for roughly 5 hours at an intensity of $\sim 1 \mathrm{~mW} / \mathrm{cm}^{2}$. The second spectrum shows a dramatic change due to exposure to light; indicating a strong light-induced change in the structural configuration of the $\mathrm{Rb}$ atom defects in the parahydrogen matrix.

The existence of multiple "trapping sites" has been long understood to be a feature of cryogenic matrices [29]. A notable exception to this rule is solid ${ }^{4} \mathrm{He}$, in which all trapping sites are equivalent $[18,30]$. IR spectra of molecules in parahydrogen indicated that the same was true for parahydrogen crystals [31]. However, Fig. 1 suggests the existence of multiple possible trapping sites for $\mathrm{Rb}$ atoms in parahydrogen: one which gives rise to absorption peaks in the $650-800 \mathrm{~nm}$ range, and others which give rise to absorption peaks in the $500-650 \mathrm{~nm}$ and $800-950 \mathrm{~nm}$ ranges. The data of Fig. 1 suggests that the application of light causes the majority of the latter trapping sites to reconfigure into the former. This is similar to what was previously observed with alkali atoms in noble gas matrices $[4,15,32]$.

More investigation is likely needed to conclusively determine how the $\mathrm{Rb}$ and parahydrogen lattice are modified by optical excitation. We speculate that the multiple trapping sites may be due to the size of the $\mathrm{Rb}$ atom [33], which is relatively large compared to the intermolecular distance of the parahydrogen crystal [34]. Consequently, it is likely that the $\mathrm{Rb}$ atoms occupy a double-substitutional site or a single-substitutional site with a vacancy next to it. Various possible orientations of double-substitutional sites would result in different excitation energies. The energy imparted to the crystal by optical excitation of the Rb atoms may allow a local "annealing" to form the most stable trapping structure.

We note that additional exposure to the light source produces negligible change in the spectrum. All subsequent data in this paper will be for crystals after exposure to the light source.

We optically pump the implanted Rb atoms using a circularly polarized laser at $755 \mathrm{~nm}$. We monitor the spin polarization of the atoms with a weak "probe beam" at the same frequency. Typical pump and probe intensities are $10^{4} \mathrm{~mW} / \mathrm{cm}^{2}$ and $10^{1} \mathrm{~mW} / \mathrm{cm}^{2}$, respectively, with beam waists on the order of $200 \mu \mathrm{m}$. After the linearlypolarized beam is sent through the sample, waveplates and a Wollaston prism are used to separate the probe into its LHC and RHC-polarized components, which are measured on two photodetectors. Both the pump and probe beams are controlled with mechanical shutters. Typically the probe is chopped at a low duty cycle to minimize its optical pumping effects.

Data from the photodetectors is shown in Fig. 2 for two different magnetic fields, after pumping by a LHC pump beam. For the intensity used, the atomic spin state is pumped on a timescale $\lesssim 10 \mathrm{~ms}$; the pumping rate is limited by the laser intensity. In Fig. 2, the crystal was pumped for a duration of $80 \mathrm{~ms}$, to saturate the polarization.

We see negligible spin polarization after optically pumping in the presence of a magnetic field which is transverse to the direction of light propagation: the absorption of LHC and RHC light is the same. This is expected, as the transverse field causes the atoms to precess rapidly, thus preventing the accumulation of significant polarization. For a longitudinal magnetic field, this is not 
the case, and we see a clear signature of spin polarization in the differential absorption of LHC and RHC light (i.e. circular dichroism).

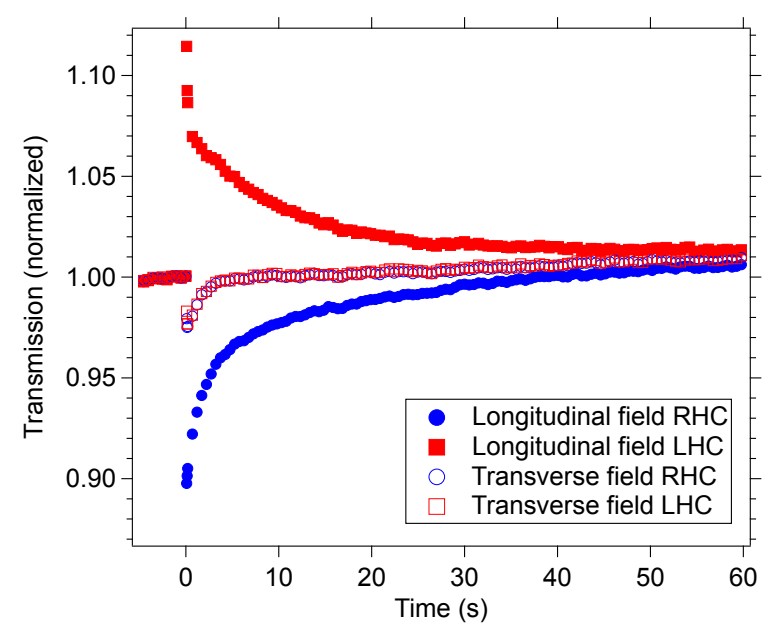

FIG. 2. (Color online) Measurement of optical pumping for a transverse field of $\sim 0.5 \mathrm{G}$ and a longitudinal field of $80 \mathrm{G}$. The crystal was optically pumped at $t=0$. The transmission signals are normalized to a level of 1 before optically pumping. This parahydrogen crystal had a Rb density of roughly $2 \times$ $10^{16} \mathrm{~cm}^{-3}$ and a peak OD of 1.5 .

Of particular note in Fig. 2 is that we observe little "bleaching" of the crystal by the pump light: the transmission of unpolarized light through the crystal is affected only slightly by the pump beam. This stands in stark contrast to earlier work examining rubidium in neon, argon, krypton, and xenon, in which the application of resonant light would severely reduce the optical depth of the crystal $[4,15,32]$.

Fig. 2 shows typical behavior for data taken after recent prior exposures of the crystal to pumping light. However, we note that the first exposure of the crystal to the pumping light decreases the OD by roughly $10 \%$. Once this occurs, additional application of pump light has negligible additional effects on the transmission, as seen in Fig. 2.

We also note that over the timescale shown in Fig. 2, there is a slight overall increase in the transmission due to optical pumping by the probe. By varying the duty cycle, we verify that - for the data presented in this paper this pumping by the probe has a negligible effect on the longitudinal relaxation time.

To extract a spin polarization signal, we calculate the ratio of the (normalized) transmission of the LHC and RHC beams, as shown in Fig. 3. The polarization signal is larger than prior work in argon by an order of magnitude [4]. However, it is still smaller than in solid He, where $50 \%$ contrast was reported [35].

The decay does not fit well to exponential decay with a single lifetime. We suspect that different trapped $\mathrm{Rb}$ atoms decay at different rates, depending on their immediate environment. As a compromise, we fit the data to the sum of two exponentials, as shown in Fig. 3, and extract two time constants for $\mathrm{T}_{1}$.

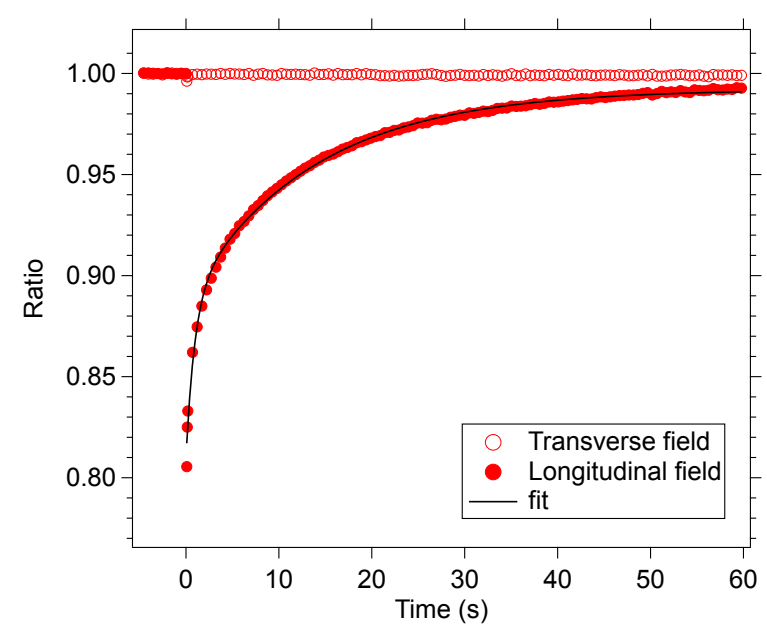

FIG. 3. (Color online) Spin polarization signal, as described in the text. The longitudinal field data is fit to a double exponential. Experimental conditions were the same as Fig. 2 .

We have measured $T_{1}$ as a function of crystal temperature, magnetic field magnitude, $\mathrm{Rb}$ density, and the parahydrogen purity of the crystal. Working with a longitudinal field of $80 \mathrm{G}$ and our lowest orthohydrogen fraction (see Supplemental Material [20]), no dependence on temperature (to within $\pm 10 \%$ ) is seen over a temperature range from 2.7 to $4.2 \mathrm{~K}$. Similarly, no dependence on $\mathrm{Rb}$ density (to within $\pm 10 \%$ ) is seen over a range from $2 \times 10^{16} \mathrm{~cm}^{-3}$ to $1 \times 10^{17} \mathrm{~cm}^{-3}$. However, $T_{1}$ shows dramatic changes with the applied field and parahydrogen purity, as discussed below.

Fig. 4 shows the time constants obtained from the double-exponential fit as a function of the longitudinal field. There is a strong dependence on the applied field, with significantly longer relaxation times at high field.

Similar behavior was observed in NV centers at cryogenic temperatures [7], in which it was attributed to "cross-relaxation" effects. This behavior was also observed in optical pumping of Cs in solid helium in the hexagonal phase [36]. In that work, the lengthening of $\mathrm{T}_{1}$ with increasing magnetic field was attributed to the Zeeman interaction decoupling the spins from their interaction with the anisotropy of their local trapping site. We speculate that similar phenomena may be happening in the parahydrogen system: spin relaxation may be driven by interaction of $\mathrm{Rb}$ with the anisotropic crystal field of the solid hydrogen matrix. By increasing the strength of the Zeeman interaction, this may effectively decouple the spin from the crystal field anisotropy. 


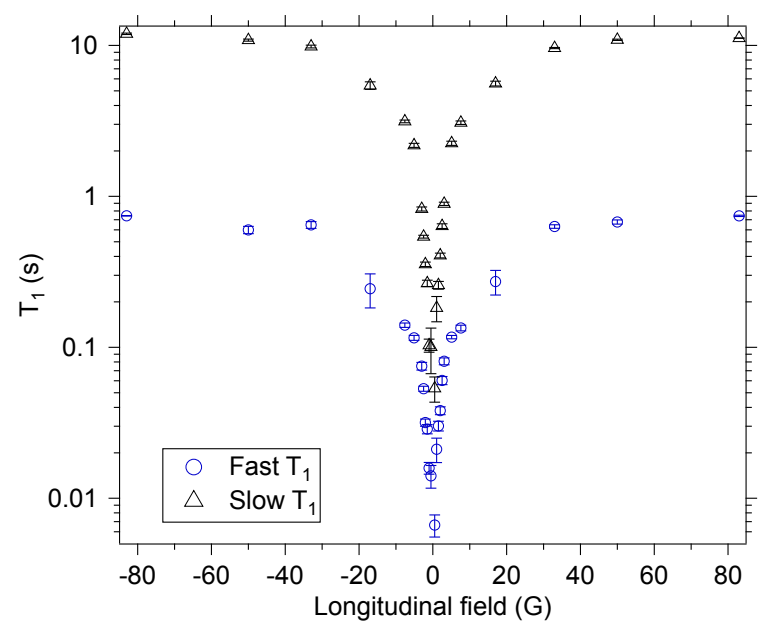

FIG. 4. (Color online) Longitudinal relaxation times as a function of the applied longitudinal magnetic field.

Fig. 5 shows the relaxation time as a function of crystal purity. To obtain this data, we have grown crystals with varying orthohydrogen fractions by varying the temperature of the ortho-para converter. The optical spectrum of the implanted $\mathrm{Rb}$ atoms shows little dependence on the orthohydrogen percentage over this range. Neither does the size of the polarization signal. However, $\mathrm{T}_{1}$ shows a strong dependence, as seen in Fig. 5.

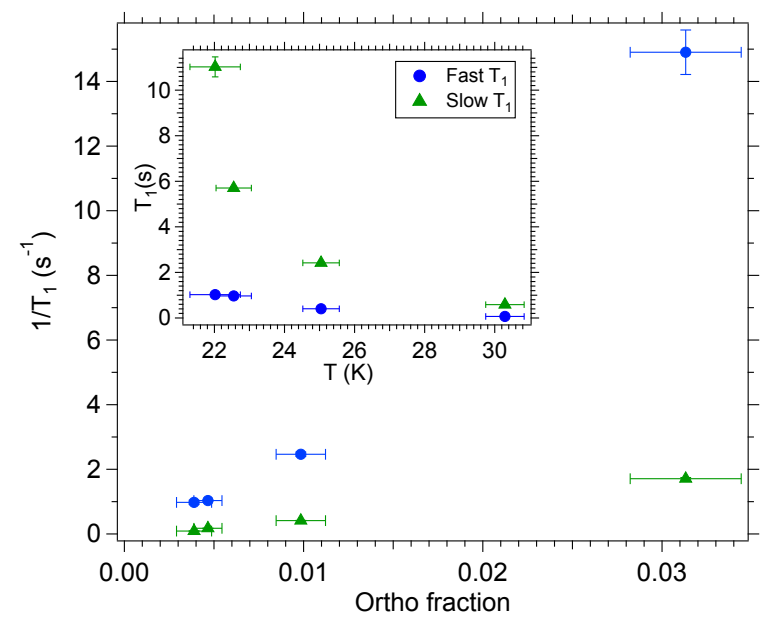

FIG. 5. (Color online) Inverse relaxation time as a function of the orthohydrogen fraction. The orthohydrogen fraction is calculated assuming the hydrogen has reached thermal equilibrium in the ortho-para converter, as discussed in the Supplemental Material [20]. The inset shows the same data, plotted as relaxation time vs. the ortho-para converter temperature.

The orthohydrogen impurities in the crystal clearly play a significant role in the relaxation time. We spec- ulate that this may be due to an increased anisotropy of the crystal field if there is a nearby orthohydrogen molecule. However, further study is needed for a conclusive understanding of this effect, as well as the field dependence.

Regardless of the mechanism, the data strongly suggests that performance superior could be achieved with parahydrogen crystals of higher purity. We note that orthohydrogen fractions $<10^{-4}$ have been achieved in prior work [37].

In conclusion, we believe that atoms implanted in solid parahydrogen host are a promising experimental platform for many applications. Even in this exploratory work, we have demonstrated $\mathrm{T}_{1}$ 's and optical state preparation and readout that are comparable to the best existing systems at comparable spin densities. We believe this system merits further exploration, including the measurement of coherence times, the preparation of crystals of higher parahydrogen purity, and the exploration of other atomic dopants.

Acknowledgements: it is our pleasure to thank Wade J. Cline and Carl D. Davison Jr. for their assistance in the construction of the apparatus. This material is based upon work supported by the National Science Foundation under Grants No. PHY 1265905 and 1607072.

*weinstein@physics.unr.edu; http://www.physics.unr.edu/xap/

[1] L. Childress, R. Walsworth, and M. Lukin, Physics Today 67, 38 (2014).

[2] V. M. Acosta, E. Bauch, M. P. Ledbetter, C. Santori, K.-M. C. Fu, P. E. Barclay, R. G. Beausoleil, H. Linget, J. F. Roch, F. Treussart, S. Chemerisov, W. Gawlik, and D. Budker, Phys. Rev. B 80, 115202 (2009).

[3] J. Maze, P. Stanwix, J. Hodges, S. Hong, J. Taylor, P. Cappellaro, L. Jiang, M. G. Dutt, E. Togan, A. Zibrov, et al., Nature 455, 644 (2008).

[4] A. N. Kanagin, S. K. Regmi, P. Pathak, and J. D. Weinstein, Physical Review A 88, 063404 (2013).

[5] M. Lemeshko, N. Y. Yao, A. V. Gorshkov, H. Weimer, S. D. Bennett, T. Momose, and S. Gopalakrishnan, Physical Review B 88, 014426 (2013).

[6] M. Arndt, S. Kanorsky, A. Weis, and T. Hänsch, Physics Letters A 174, 298 (1993).

[7] A. Jarmola, V. M. Acosta, K. Jensen, S. Chemerisov, and D. Budker, Phys. Rev. Lett. 108, 197601 (2012).

[8] A. M. Tyryshkin, S. Tojo, J. J. Morton, H. Riemann, N. V. Abrosimov, P. Becker, H.-J. Pohl, T. Schenkel, M. L. Thewalt, K. M. Itoh, et al., Nature materials 11, 143 (2012).

[9] H. Büch, S. Mahapatra, R. Rahman, A. Morello, and M. Simmons, Nature communications 4 (2013).

[10] G. Feher and E. Gere, Physical Review 114, 1245 (1959).

[11] E. Fraval, M. Sellars, and J. Longdell, Physical review letters 95, 030506 (2005).

[12] F. Bussières, N. Sangouard, M. Afzelius, H. de Riedmatten, C. Simon, and W. Tittel, Journal of Modern Optics 
60, 1519 (2013).

[13] M. Romalis and M. Ledbetter, Physical review letters 87, 067601 (2001).

[14] C.-Y. Xu, S.-M. Hu, J. Singh, K. Bailey, Z.-T. Lu, P. Mueller, T. P. O'Connor, and U. Welp, Phys. Rev. Lett. 107, 093001 (2011).

[15] P. Pathak, Absorption Spectrum of Rubidium in a Solid Neon Matrix, M.S. Thesis, University of Nevada, Reno (2014).

[16] M. Arndt, S. I. Kanorsky, A. Weis, and T. W. Hänsch, Phys. Rev. Lett. 74, 1359 (1995).

[17] S. I. Kanorsky, S. Lang, S. Lücke, S. B. Ross, T. W. Hänsch, and A. Weis, Phys. Rev. A 54, R1010 (1996).

[18] P. Moroshkin, A. Hofer, S. Ulzega, and A. Weis, Low Temperature Physics 32, 981 (2006).

[19] T. Furukawa, Y. Matsuo, A. Hatakeyama, Y. Fukuyama, T. Kobayashi, H. Izumi, and T. Shimoda, Phys. Rev. Lett. 96, 095301 (2006).

[20] See Supplemental Material at [URL will be inserted by publisher] for a description of the growth and measurement of our parahydrogen crystals.

[21] S. Tam and M. E. Fajardo, Review of Scientific Instruments 70, 1926 (1999).

[22] A. N. Kanagin, Creation and Analysis of Para-Hydrogen Crystals, B.S. Thesis, University of Nevada, Reno (2015).

[23] S. Aldrich, Iron (III) Oxide; hydrated, catalyst grade, 30-50 mesh.

[24] Cryomech, PT405.

[25] M. Perera, B. A. Tom, Y. Miyamoto, M. W. Porambo, L. E. Moore, W. R. Evans, T. Momose, and B. J. McCall, Optics letters 36, 840 (2011).

[26] J. Ekin, Experimental Techniques for Low-Temperature
Measurements: Cryostat Design, Material Properties and Superconductor Critical-Current Testing: Cryostat Design, Material Properties and Superconductor CriticalCurrent Testing (OUP Oxford, 2006).

[27] I. F. Silvera, Rev. Mod. Phys. 52, 393 (1980).

[28] C. Hartzell, Matrix Isolation of Rubidium in a Solid Para-Hydrogen Substrate, B.S. Thesis, University of Nevada, Reno (2014).

[29] S. L. Kupferman and F. M. Pipkin, Phys. Rev. 166, 207 (1968).

[30] P. Moroshkin, A. Hofer, and A. Weis, Physics Reports 469, 1 (2008).

[31] T. Momose, H. Hoshina, M. Fushitani, and H. Katsuki, Vibrational spectroscopy 34, 95 (2004).

[32] I. Gerhardt, K. Sin, and T. Momose, The Journal of chemical physics 137, 014507 (2012).

[33] M. Mantina, A. C. Chamberlin, R. Valero, C. J. Cramer, and D. G. Truhlar, The Journal of Physical Chemistry A 113, 5806 (2009).

[34] T. Momose and T. Shida, Bulletin of the Chemical Society of Japan 71, 1 (1998).

[35] A. Weis, S. Kanorsky, M. Arndt, and T. Hänsch, Zeitschrift für Physik B Condensed Matter 98, 359 (1995).

[36] S. Lang, S. Kanorsky, T. Eichler, R. Müller-Siebert, T. W. Hänsch, and A. Weis, Phys. Rev. A 60, 3867 (1999).

[37] B. A. Tom, S. Bhasker, Y. Miyamoto, T. Momose, and B. J. McCall, Review of Scientific Instruments 80, 016108 (2009). 